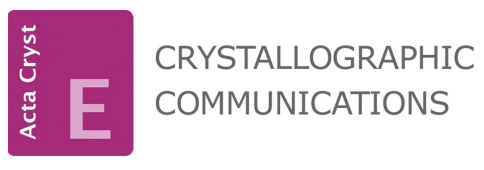

ISSN 2056-9890

Received 16 August 2017

Accepted 9 November 2017

Edited by W. Imhof, University Koblenz-Landau, Germany

Keywords: palladium-amino acid complex; Lserine; single-crystal $\mathrm{X}$-ray structure; palladium complex.

CCDC reference: 1584689

Supporting information: this article has supporting information at journals.iucr.org/e

\section{Crystal structure of poly[[ $\mu_{3}-(S)-2$-amino-3- hydroxypropanoato]-cis-di- $\mu$-chlorido-caesium- palladium(II)]}

\author{
Mohan Madhav Bhadbhade $^{\mathrm{a}}$ and Alexander J. Charlson ${ }^{\mathrm{b} *}$ \\ ${ }^{\mathbf{a}}$ Mark Wainwright Analytical Centre, University of New South Wales, Sydney, NSW 2052, Australia, and ${ }^{\mathbf{b}}$ Department of \\ Chemistry and Biomolecular Sciences, Macquarie University, North Ryde, NSW 2109, Australia. *Correspondence \\ e-mail: alec.charlson@gmail.com
}

The structure of the title compound, $\left[\mathrm{CsPd}\left(\mathrm{C}_{3} \mathrm{H}_{6} \mathrm{NO}_{3}\right) \mathrm{Cl}_{2}\right]_{n}$, previously shown to have anticancer activity in rodent test systems and recently found to have antifungal activity, has been determined. The Pd centre is in a square-planar coordination environment with two chlorine atoms in cis positions and the remaining two coordination sites being coordinated by $\mathrm{N}$ and $\mathrm{O}$ atoms from deprotonated L-serine. Each of the Cs cations shows ninefold coordination with six chlorine and three $\mathrm{O}$ atoms resulting in a coordination environment that is similar to the well known $\mathrm{Cs}_{2} \mathrm{SO}_{4}$ structure. X-ray crystal structures of only three dichloridopalladium(II)-amino acid complexes have been determined so far and the present paper describes one of those.

\section{Chemical context}

The X-ray crystal structure of potassium-L-alaninato-dichloroplatinate(II) has been published (Schiesser et al., 2012). Two complexes of L-serine with palladium(II), bis(L-serinato) palladium(II) and caesium cis-dichloro-L-serinato palladium(II), were synthesized (Charlson et al., 1981) and an $\mathrm{X}$-ray crystal structure determination of bis (L-serinato) palladium(II) has been performed (Vagg, 1979). Previously it was shown that caesium cis-dichloro-L-serinato palladium(II) produced filamentous growth in Escherichia coli (E.coli) bacteria (Charlson et al., 1981), markedly modified the interior of E.coli bacteria cells (McArdle et al., 1984), increased the lifespan of solid murine tumors Ca-755 and RShM-5 (Treschalina et al., 1994) and had radio-modifying properties (Treshalina et al., 1995). Recently it was found that caesium cis-dichloro-serinato palladium(II) had antifungal activity in the Candida albicans and Cryptococcus neoformans testsystems and was non-cytotoxic against human kidney cells at the dose levels used (Elliott, 2016). The antimicrobial screening was performed by CO-ADD (The Community for Antimicrobial Drug Discovery) funded by the Welcome Trust (UK) and the University of Queensland (Australia). In the publication describing the synthesis of caesium cis-dichloro-Lserinato palladium(II), the empirical formula of the compound was deduced on the basis of the percentages of carbon, hydrogen, chlorine and nitrogen that were obtained by micro analysis (Charlson et al., 1981) The present X-ray crystal structure was performed in order to establish the molecular and structural formulae of caesium cis-dichloro-Lserinato palladium(II). 
<smiles>C[C@@]1(CO)N[Pb](Cl)(Cl)OC1=O</smiles>

\section{Structural commentary}

The palladium(II) serine complex ion shows a square-planar coordination of palladium with the two chloro ligands being in cis positions relative to each other and the remaining two coordination sites being coordinated by the nitrogen atom (N1) and one of the carboxylato oxygen atoms (O1) of the deprotonated amino acid L-serine. The view of the asymmetric unit is given in Fig. 1 and the ninefold coordination (three oxygen and six chlorine atoms) of caesium is shown in Fig. 2. A summary of significant bond distances is given in Table 1 . The two $\mathrm{Pd}-\mathrm{Cl}$ bonds are of slightly different bond length. The longer bond $[\mathrm{Pd} 1-\mathrm{Cl} 1=2.305(4) \mathrm{A}]$ is trans to nitrogen and the shorter one $[\mathrm{Pd} 1-\mathrm{Cl} 2=2.287$ (4) A] is trans to the oxygen atom. The same behaviour was observed in the structure of barium dichloro(glycinato) palladium(II) $2 \mathrm{H}_{2} \mathrm{O}$ (Baidina et al., 1980a). The five membered ring $\mathrm{Pd} 1-\mathrm{O} 1-\mathrm{C} 1-\mathrm{C} 2-\mathrm{N} 1$ is planar with the hydroxymethyl substituent in a gauche-gauche orientation that is very similar with the conformation of one of the ligands in the structure of bis(L-serinato) palladium(II) (Vagg, 1979).

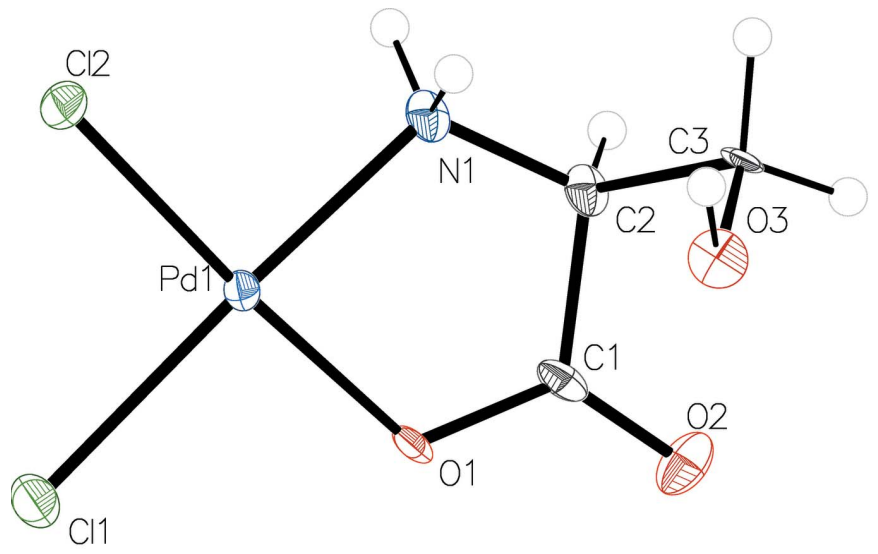

Figure 1

ORTEP representation of the asymmetric unit showing atom labelling (ellipsoids drawn at $50 \%$ probabilities).

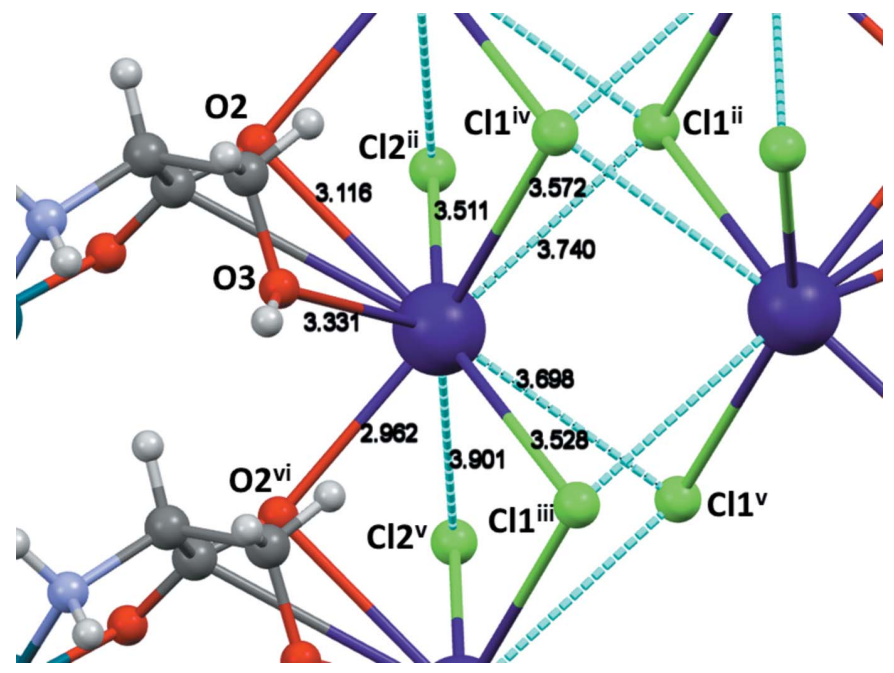

Figure 2

Caesium coordination and packing of cations and anions in the unit cell.

\section{Supramolecular features}

The cation and anion assembly, viewed along the twofold axis (the $c$ axis) is shown in Fig. 3. Chains of complex anions related by a $21_{1}$ screw axis along the $b$ axis link double rows of caesium cations (Fig. 4). The caesium ions are bridged by chlorine atoms along and across the rows. The successful crystallization with larger Cs ions, which failed with smaller K ions, can be rationalized with this lattice arrangement. Larger cations with higher coordination capability can engage four molecules of complex anions acting as a nucleator in forming the lattice much better compared to smaller cations such as $\mathrm{Na}$ or $\mathrm{Li}$.

In the crystal, extensive $\mathrm{O}-\mathrm{H} \cdots \mathrm{O}, \mathrm{N}-\mathrm{H} \cdots \mathrm{Cl}$ and $\mathrm{C}-$ $\mathrm{H}$... O hydrogen bonds (Table 2, Fig. 5) link the molecules, forming a two-dimensional network parallel to (010).

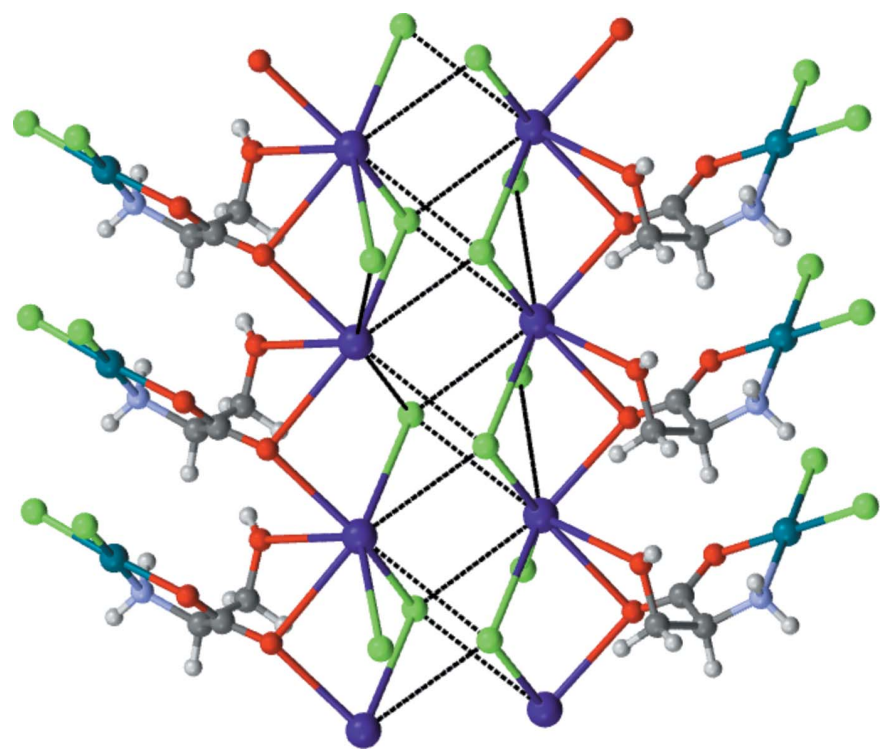

Figure 3

The cation and anion assembly viewed along the twofold axis (the $c$ axis). 
Table 1

Selected bond lengths $(\AA)$.

\begin{tabular}{llll}
\hline $\mathrm{Cs} 1-\mathrm{Cs} 1^{\mathrm{i}}$ & $4.421(2)$ & $\mathrm{Cs} 1-\mathrm{O} 2$ & $3.117(10)$ \\
$\mathrm{Cs} 1-\mathrm{Pd} 1^{\mathrm{ii}}$ & $3.8755(17)$ & $\mathrm{Cs} 1-\mathrm{O} 2^{\mathrm{vi}}$ & $2.962(10)$ \\
$\mathrm{Cs} 1-\mathrm{Cl} 1^{\mathrm{iii}}$ & $3.528(4)$ & $\mathrm{Cs} 1-\mathrm{O} 3$ & $3.349(10)$ \\
$\mathrm{Cs} 1-\mathrm{Cl} 1^{\text {iv }}$ & $3.572(4)$ & $\mathrm{Cs} 1-\mathrm{C} 1$ & $3.654(15)$ \\
$\mathrm{Cs} 1-\mathrm{Cl} 1^{\mathrm{ii}}$ & $3.740(4)$ & $\mathrm{Pd} 1-\mathrm{Cl} 1$ & $2.305(4)$ \\
$\mathrm{Cs} 1-\mathrm{Cl} 1^{\mathrm{v}}$ & $3.698(4)$ & $\mathrm{Pd} 1-\mathrm{Cl} 2$ & $2.287(4)$ \\
$\mathrm{Cs} 1-\mathrm{Cl} 2^{\mathrm{ii}}$ & $3.510(4)$ & $\mathrm{Pd} 1-\mathrm{O} 1$ & $1.993(9)$ \\
$\mathrm{Cs} 1-\mathrm{Cl} 2^{\mathrm{v}}$ & $3.901(4)$ & $\mathrm{Pd} 1-\mathrm{N} 1$ & $2.000(12)$ \\
\hline
\end{tabular}

Symmetry codes: (i) $-x+1,-y, z$; (ii) $-x+\frac{1}{2}, y-\frac{1}{2},-z+1$; (iii) $x+\frac{1}{2},-y+\frac{1}{2},-z+2$; (iv) $x+\frac{1}{2},-y+\frac{1}{2},-z+1$; (v) $-x+\frac{1}{2}, y-\frac{1}{2},-z+2$; (vi) $x, y, z+1$.

\section{Database survey}

The planarity of the chelate ring $M-\mathrm{N}-\mathrm{CH}(R)-\mathrm{C}-\mathrm{O}$ is thought to be a relevant structural parameter in correlating biological activities and was examined in the structures of Pd (36 hits) and Pt (49 hits) complexes from the Cambridge Structural Database (CSD; Groom et al. 2016) using CONQUEST (Version 1.19; Bruno et al., 2002). However, there are very few structure determinations of $\mathrm{Pd}$ or $\mathrm{Pt}$ complexes with amino acids as the organic ligands, only five having been reported for $\mathrm{Pt}$ and three for Pd. Table 3 details these structures and their chelate ring geometry parameters in relation to their planarity. It appears from Table 3 that the planarity of the fivemembered ring is dependent on the hybridization state of the carboxylate moiety after it has coordinated to the metal ion. Thus longer $\mathrm{C}-\mathrm{O}$ bonds (associated with shorter exocyclic ones) give rise to larger $\mathrm{O}-\mathrm{C}-\mathrm{C}-\mathrm{N}$ torsion angles (and non-planarity), whereas more equal $\mathrm{C}-\mathrm{O}$ bonds form planar five-membered rings. For example, structure ACEMEC (Schiesser et al., 2012) has the highest torsion angle $\left(25.85^{\circ}\right)$ accompanied by a quite long $\mathrm{C}-\mathrm{O}$ bond length $(1.304 \AA$ )

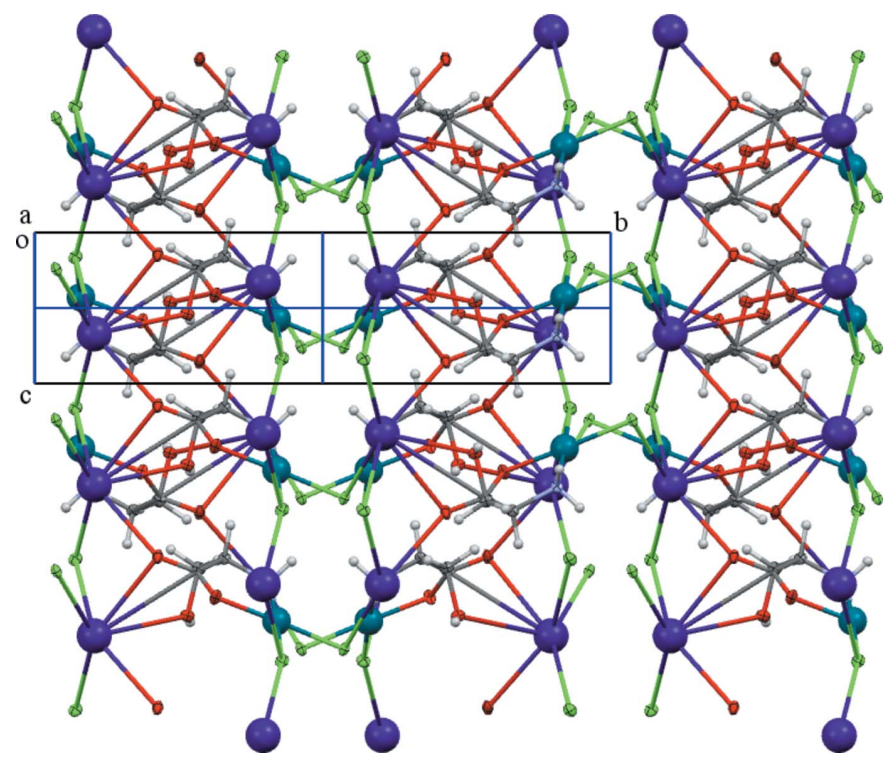

Figure 4

Chains of complex anions related by a $2_{1}$ screw axis along the $a$ axis linking double rows of caesium cations (blue spheres).
Table 2

Hydrogen-bond geometry $\left(\AA{ }^{\circ}\right)$.

\begin{tabular}{lllll}
\hline$D-\mathrm{H} \cdots A$ & $D-\mathrm{H}$ & $\mathrm{H} \cdots A$ & $D \cdots A$ & $D-\mathrm{H} \cdots A$ \\
\hline $\mathrm{O} 3-\mathrm{H} 3 \cdots \mathrm{O} 1^{\text {iv }}$ & $0.91(1)$ & $2.07(2)$ & $2.750(14)$ & $131(3)$ \\
$\mathrm{O} 3-\mathrm{H} 3 \cdots 2^{\text {iv }}$ & $0.91(1)$ & $2.60(2)$ & $3.479(15)$ & $163(3)$ \\
$\mathrm{N} 1-\mathrm{H} 1 A \cdots \mathrm{C} 2^{\text {vii }}$ & 0.91 & 2.62 & $3.471(13)$ & 156 \\
$\mathrm{~N} 1-\mathrm{H} 1 B \cdots \mathrm{C} 2^{\text {viii }}$ & 0.91 & 2.51 & $3.388(13)$ & 163 \\
$\mathrm{C} 2-\mathrm{H} 2 \cdots 3^{\text {vii }}$ & 1.00 & 2.58 & $3.255(19)$ & 125 \\
\hline
\end{tabular}

Symmetry codes: (iv) $x+\frac{1}{2},-y+\frac{1}{2},-z+1$; (vii) $x, y, z-1$; (viii) $-x+1,-y+1, z$

whereas structure BAGLPD (Baidina et al., 1980a) shows the smallest torsion angle $\left(5.36^{\circ}\right)$ together with a slightly shorter C-O bond length (1.284 $\AA$ ). Irrespective of its causes (electronic factors during the complex formation), this parameter could be an important feature while modelling the interaction of the complexes with DNA for biological activities.

\section{Biological considerations}

Caesium cis-dichloro-L-serinato platinum(II) has been shown to increase the lifespan of P-388 leukemic mice. It also has anti-tumor activity in the MBG5 Supernal Capsule MX-1 mammary carcinoma xenograph mouse test-system (Charlson \& Shorland, 1984). An X-ray crystal structure determination of caesium cis-dichloro-L-serinato platinum(II) has not been performed. Since caesium cis-dichloro-L-serinato platinum(II) and caesium cis-dichloro-L-serinato palladium(II) both show anticancer activity in mouse test-systems, it may be anticipated that the platinum(II) complex also has a planar fivemembered ring system. Recently, there has been a report on the structure of potassium (2-amino-3-hydroxypropanoato)dichloroplatinum(II) (Fabbiani et al., 2015) in which one molecule has a planar ring. Potassium cis-dichloro-glycinato platinum(II) has also been shown to increase the lifespan of

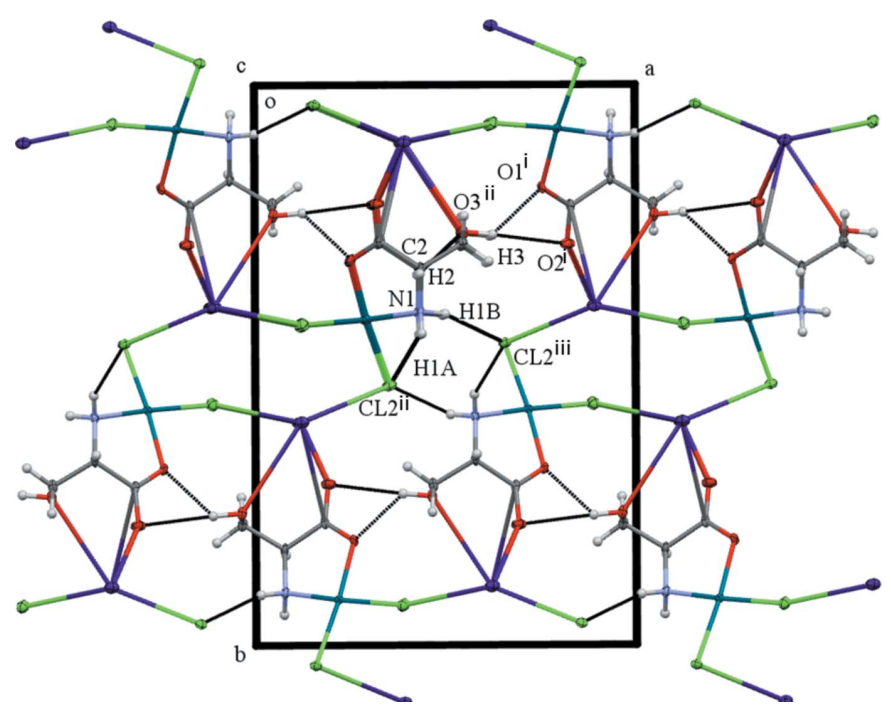

Figure 5

A view of the packing illustrating the hydrogen bonding (dashed lines; see Table 2). 
Table 3

Molecular geometry $\left(\AA{ }^{\circ}\right)$ of the five-membered ring in $M \mathrm{Cl}_{2}$ (amino acid) complexes with Pt and Pd.

Data obtained from a search of the CSD (Groom et al., 2016).

\begin{tabular}{|c|c|c|c|c|c|c|c|}
\hline CCDC refcode & Reference & Structure & $\mathrm{C}-\mathrm{O}$ & $\mathrm{C}=\mathrm{O}$ & $\mathrm{C}-\mathrm{C}$ & $\mathrm{C}-\mathrm{N}$ & $\mathrm{O}-\mathrm{C}-\mathrm{C}-\mathrm{N}(\tau)$ \\
\hline ACEMEC & Schiesser et al., (2012) & $\mathrm{K}\left[\mathrm{Pt}(\mathrm{L}-\mathrm{alaO}) \mathrm{Cl}_{2}\right]$ & 1.304 & 1.223 & 1.528 & 1.480 & 25.85 \\
\hline GAWYOS & Bino et al., (1988) & {$\left[\mathrm{PtCl}_{2}(\mathrm{~N}, \mathrm{O}-\mathrm{Dap})\right]$} & 1.313 & 1.232 & 1.543 & 1.499 & 19.44 \\
\hline GAWYUY & Bino et al., (1988) & {$\left[\mathrm{PtCl}_{2}(\mathrm{~N}, \mathrm{O}\right.$-Lys $\left.)\right] \cdot \mathrm{H}_{2} \mathrm{O}$} & 1.300 & 1.219 & 1.500 & 1.557 & 13.66 \\
\hline KCGLPD & Baidina et al., (1980b) & $\mathrm{K}\left[\mathrm{Pd}(\mathrm{Gly}) \mathrm{Cl}_{2}\right] \cdot \mathrm{H}_{2} \mathrm{O}$ & 1.285 & 1.216 & 1.518 & 1.490 & 11.74 \\
\hline BAGLPD & Baidina et al., (1980a) & $\mathrm{Ba}\left[\mathrm{Pd}(\mathrm{Gly}) \mathrm{Cl}_{2}\right] \cdot 2 \mathrm{H}_{2} \mathrm{O}$ & 1.268 & 1.194 & 1.526 & 1.484 & -13.69 \\
\hline BAGLPD & Baidina et al., $(1980 a)$ & $\mathrm{Ba}\left[\mathrm{Pd}(\mathrm{Gly}) \mathrm{Cl}_{2}\right] \cdot 2 \mathrm{H}_{2} \mathrm{O}$ & 1.284 & 1.233 & 1.503 & 1.507 & 5.36 \\
\hline
\end{tabular}

P-388 leukemic mice (Charlson \& Shorland, 1984). Therefore the hydroxyl group in caesium cis-dichloro-L-serinato platinum(II) plays little or no part in the anti-tumor activity shown by this complex. In the publication by Schiesser et al. (2012), the authors mentioned that some platinum(II) complexes with amino acid ligands showed moderate cytotoxicity toward tumor cells. However, they did not mention whether potassium-L-alaninato-dichloro platinum(II) has been tested or not in any of the rodent test-systems. It should also be mentioned that potassium cis-dichloroglycinato platinum(II) and caesium cis-dichloro-L-serinato platinum(II) have not been screened for possible antifungal activity. The X-ray crystal structure of bis(phenylglycinato)palladium(II) containing two molecules of dimethyl sulfoxide has been determined (Gao et al., 2009). These authors also synthesized bis(phenylglycinato)platinum(II), which also contains two molecules of dimethyl sulfoxide, and showed that this platinum complex had a stronger binding affinity to fish-sperm DNA than the corresponding palladium complex. Both complexes added to DNA by a strong intercalating mode and both complexes could cleave pBR 332plasmid DNA. (A plasmid is a small DNA molecule within a cell that is physically separated from chromosomal DNA and replicates independently. Plasmids are commonly found in bacteria as circular double-stranded DNA. Plasmid DNA can also be found in fungi and higher plants.) The palladium and platinum complexes are also cytotoxic to HeLa, Hep-G2, KB, and AGZY-83a tumor cells, with the platinum complex being more effective than the palladium complex. X-ray crystal structures have been reported for the palladium(II) complexes of glycine with 2,2'-bipyridine, 1,10-phenathroline or 2,2'-bipyridylamine with chloride counter-ions (Yodoshi \& Okabe, 2008). Each of the complexes was shown to be capable of intercalative binding to calf thymus DNA and could enhance the cleavage of pBR 332 plasmid DNA in the presence of hydrogen peroxide and ascorbic acid (Yodoshi \& Okabi, 2008). Small molecules can intercalate DNA by fitting in between base pairs in the two different DNA strands. Generally these molecules are planar or nearly planar. In the case of the palladium(II) complex with glycine and bipyridine, the central palladium(II) atom has a distorted square-planar geometry. Furthermore, the two fivemembered rings formed by the bipyridine and the glycine ligands are almost planar and the two pyridine rings are planar (Yodoshi \& Okabe, 2008).

\section{Synthesis and crystallization}

Poly\{caesium [cis-dichloro-(S-2-amino-3-hydroxypropanoate$\kappa^{2} N, O$ )palladate(II)]\} was synthesized by a previously described method (Charlson et al., 1981). Using a procedure similar to the method described for the synthesis of potassiumL-alaninato-dichloroplatinum(II) (Ley \& Ficken, 1912), a crude amorphous sample of potassium cis-dichloro-L-serinato palladium(II) was obtained. Therefore, the potassium salt was converted by a known method (Cleare, 1977) into crystalline caesium cis-dichloro-L-serinato palladium(II), which could be purified by recrystallization from water. In a typical prepara-

Table 4

Experimental details.

\begin{tabular}{|c|c|}
\hline \multicolumn{2}{|l|}{ Crystal data } \\
\hline Chemical formula & {$\left[\mathrm{CsPd}\left(\mathrm{C}_{3} \mathrm{H}_{6} \mathrm{NO}_{3}\right) \mathrm{Cl}_{2}\right]$} \\
\hline$M_{\mathrm{r}}$ & 414.30 \\
\hline Crystal system, space group & Orthorhombic, $P 2_{1} 2_{1} 2$ \\
\hline Temperature $(\mathrm{K})$ & 150 \\
\hline$a, b, c(\AA)$ & $11.594(4), 17.072(5), 4.4739$ (12) \\
\hline$V\left(\AA^{3}\right)$ & $885.6(5)$ \\
\hline$Z$ & 4 \\
\hline Radiation type & Mo $K \alpha$ \\
\hline$\mu\left(\mathrm{mm}^{-1}\right)$ & 6.71 \\
\hline Crystal size $(\mathrm{mm})$ & $0.08 \times 0.07 \times 0.03$ \\
\hline \multicolumn{2}{|l|}{ Data collection } \\
\hline Diffractometer & Bruker APEXII CCD \\
\hline Absorption correction & $\begin{array}{l}\text { Multi-scan (SADABS; Bruker, } \\
\text { 2016) }\end{array}$ \\
\hline$T_{\min }, T_{\max }$ & $0.499,0.746$ \\
\hline $\begin{array}{l}\text { No. of measured, independent and } \\
\text { observed }[I>2 \sigma(I)] \text { reflections }\end{array}$ & $5126,1546,1212$ \\
\hline$R_{\text {int }}$ & 0.125 \\
\hline$(\sin \theta / \lambda)_{\max }\left(\AA^{-1}\right)$ & 0.595 \\
\hline \multicolumn{2}{|l|}{ Refinement } \\
\hline$R\left[F^{2}>2 \sigma\left(F^{2}\right)\right], w R\left(F^{2}\right), S$ & $0.039,0.076,0.83$ \\
\hline No. of reflections & 1546 \\
\hline No. of parameters & 91 \\
\hline No. of restraints & 13 \\
\hline $\mathrm{H}$-atom treatment & $\begin{array}{l}\mathrm{H} \text { atoms treated by a mixture of } \\
\text { independent and constrained } \\
\text { refinement }\end{array}$ \\
\hline$\Delta \rho_{\max }, \Delta \rho_{\min }\left(\mathrm{e} \AA^{-3}\right)$ & $1.64,-1.24$ \\
\hline Absolute structure & $\begin{array}{l}\text { Flack } x \text { determined using } 375 \\
\text { quotients }\left[\left(I^{+}\right)-\left(I^{-}\right)\right] /\left[\left(I^{+}\right)+\left(I^{-}\right)\right] \\
\text {(Parsons } \text { et al., } 2013)\end{array}$ \\
\hline Absolute structure parameter & $-0.02(5)$ \\
\hline
\end{tabular}

Computer programs: APEX2 and SAINT (Bruker, 2016), SHELXT (Sheldrick, 2015a), SHELXL2014 (Sheldrick, 2015b) and OLEX2 (Dolomanov et al., 2009). 
tion, a solution of L-serine $(2.1 \mathrm{~g})$ and potassium tetrachloropalladate(II) (3.2 g) in water $(60 \mathrm{~mL})$ was heated for $3 \mathrm{~h}$ under reflux on a boiling water bath. Absolute ethanol $(450 \mathrm{~mL})$ was added to the filtered reaction mixture and the light-orange precipitate $(1.7 \mathrm{~g})$ was filtered off. This potassium salt of the palladium L-serine complex was reprecipitated from water $(10 \mathrm{~mL})$ with ethanol $(40 \mathrm{~mL})$. Small quantities of solid caesium chloride were added to a stirred solution of the potassium salt $(1.5 \mathrm{~g})$ in water $(10 \mathrm{~mL})$ until the solution became dark red. A brick-shaped red crystalline caesium salt $(1.2 \mathrm{~g})$ was obtained by keeping this solution for $24 \mathrm{~h}$ at $278 \mathrm{~K}$. The caesium complex was purified by two recrystallizations from water (yield $0.2 \mathrm{~g}$ ). Analysis found: C, 8.83; H, 1.55; Cl, 17.2; N, 3.43. Calculated for $\mathrm{C}_{3} \mathrm{H}_{6} \mathrm{Cl}_{2} \mathrm{NO}_{3} \mathrm{PdCs}$ : C, 8.70; $\mathrm{H}, 1.46$; $\mathrm{Cl}, 17.1$; N, 3.38\%.

\section{Refinement}

Crystal data, data collection and structure refinement details are summarized in Table 4. All $\mathrm{H}$ atoms were positioned geometrically with $d(\mathrm{~N}-\mathrm{H})=0.91 \AA$, for $\mathrm{Csp} p^{3}-\mathrm{H}, d(\mathrm{C}-\mathrm{H})$ $=0.99 \AA$ and $(\mathrm{O}-\mathrm{H})=0.87 \AA$ and with $U_{\text {iso }}(\mathrm{H})=1.2 U_{\text {eq }}(\mathrm{C}, \mathrm{N})$ or $1.5 U_{\text {eq }}(\mathrm{O})$.

\section{Acknowledgements}

We thank the Johnson and Matthey Company in Reading, England, for supplying the potassium tetrachloropalladate(II) that was used in the preparation of caesium cis-dichloro-Lserinatopalladium(II) on their loan scheme. We also thank Dr Alysha Elliott from the CO-ADD of the University of Queensland for giving us the results of the antifungal testing and Dr Andrew Piggott of the Department of Chemistry and Biological Sciences for taking an interest in this work. In addition, we thank Dr Christopher Marjo, Head of the Division (SSEAU), Mark Wainwright Analytical Centre, UNSW for his encouragement and support.

\section{References}

Baidina, I. A., Podberezskaya, N. V. \& Borisov, S. V. (1980a). Zh. Strukt. Khim. 21, 119-125.

Baidina, I. A., Podberezskaya, N. V. \& Borisov, S. V. (1980b). Zh. Strukt. Khim. 21, 198-203.

Bino, A., Cohen, S., Altman, J. \& Wilchek, M. (1988). Inorg. Chim. Acta, 147, 99-102.

Bruker (2016). APEX2, SAINT and SADABS. Bruker AXS Inc., Madison, Wisconsin, USA.

Bruno, I. J., Cole, J. C., Edgington, P. R., Kessler, M., Macrae, C. F., McCabe, P., Pearson, J. \& Taylor, R. (2002). Acta Cryst. B58, 389397.

Charlson, A. J., McArdle, N. T. \& Watton, E. C. (1981). Inorg. Chim. Acta, 56, L35-L36.

Charlson, A. J. \& Shorland, W. A. (1984). Inorg. Chim. Acta, 93, L67L68.

Cleare, M. J. (1977). SAE J.-Automot. Eng. Tech. Pap. 1, 770061.

Dolomanov, O. V., Bourhis, L. J., Gildea, R. J., Howard, J. A. K. \& Puschmann, H. (2009). J. Appl. Cryst. 42, 339-341.

Elliott, A. (2016). Personal communication. CO-ADD, University of Queensland, Australia.

Fabbiani, F., Sadler, P. \& Zobi, F. (2015). Private communication (refcode 1413050). CCDC, Cambridge, England.

Gao, E., Wu, Q., Wang, C., Zhu, M., Wang, L., Liu, H., Huang, Y. \& Sun, Y. (2009). J. Coord. Chem. 62, 3425-3437.

Groom, C. R., Bruno, I. J., Lightfoot, M. P. \& Ward, S. C. (2016). Acta Cryst. B72, 171-179.

Ley, H. \& Ficken, F. (1912). Ber. Dtsch. Chem. Ges. 45, 377-382.

McArdle, N. T., Charlson, A. J., Shorey, C. D., Arnold, R. \& Barker, N. (1984). Inorg. Chim. Acta, 92, 113-121.

Parsons, S., Flack, H. D. \& Wagner, T. (2013). Acta Cryst. B69, 249 259.

Schiesser, S., Mayer, P., Carell, T. \& Beck, W. (2012). Z. Naturforsch. Teil B, 67, 849-852.

Sheldrick, G. M. (2015a). Acta Cryst. A71, 3-8.

Sheldrick, G. M. (2015b). Acta Cryst. C71, 3-8.

Treschalina, H. M., Charlson, A. J., Shorland, W. A., Sedakova, L. A. \& Firsova, G. A. (1994). Herald Cancer Res. Centre AMS Russia, 2 , 28-32.

Treshalina, H. M., Krimker, V. M., Charlson, A. J. \& Shorland, W. A. (1995). Azerbaijan J. Oncol. Relat. Sci. 1, 62-63.

Vagg, R. S. (1979). Acta Cryst. B35, 341-344.

Yodoshi, M. \& Okabe, N. (2008). Chem. Pharm. Bull. 56, 908-914. 


\section{supporting information}

Acta Cryst. (2017). E73, 1898-1902 [https://doi.org/10.1107/S2056989017016164]

Crystal structure of poly[[$\mu_{3}-(S)$-2-amino-3-hydroxypropanoato]-cis-di- $\mu$ chlorido-caesiumpalladium(II)]

\section{Mohan Madhav Bhadbhade and Alexander J. Charlson}

\section{Computing details}

Data collection: APEX2 (Bruker, 2016); cell refinement: SAINT (Bruker, 2016); data reduction: SAINT (Bruker, 2016); program(s) used to solve structure: SHELXT (Sheldrick, 2015a); program(s) used to refine structure: SHELXL2014 (Sheldrick, 2015b); molecular graphics: OLEX2 (Dolomanov et al., 2009); software used to prepare material for publication: OLEX2 (Dolomanov et al., 2009).

Poly[[ $\mu_{3}-(S)$-2-amino-3-hydroxypropanoato]-cis-di- $\mu$-chlorido-caesiumpalladium(II)]

\section{Crystal data}

$\left[\mathrm{CsPd}\left(\mathrm{C}_{3} \mathrm{H}_{6} \mathrm{NO}_{3}\right) \mathrm{Cl}_{2}\right]$

$M_{r}=414.30$

Orthorhombic, $P 22_{1} 2_{1} 2$

$a=11.594(4) \AA$

$b=17.072(5) \AA$

$c=4.4739(12) \AA$

$V=885.6(5) \AA^{3}$

$Z=4$

$F(000)=760$

Data collection

Bruker APEXII CCD

diffractometer

$\varphi$ and $\omega$ scans

Absorption correction: multi-scan

(SADABS; Bruker, 2016)

$T_{\min }=0.499, T_{\max }=0.746$

5126 measured reflections

Refinement

Refinement on $F^{2}$

Least-squares matrix: full

$R\left[F^{2}>2 \sigma\left(F^{2}\right)\right]=0.039$

$w R\left(F^{2}\right)=0.076$

$S=0.83$

1546 reflections

91 parameters

13 restraints

Hydrogen site location: mixed
$D_{\mathrm{x}}=3.107 \mathrm{Mg} \mathrm{m}^{-3}$

Mo $K \alpha$ radiation, $\lambda=0.71073 \AA$

Cell parameters from 360 reflections

$\theta=2.4-20.1^{\circ}$

$\mu=6.71 \mathrm{~mm}^{-1}$

$T=150 \mathrm{~K}$

Plate, light yellow

$0.08 \times 0.07 \times 0.03 \mathrm{~mm}$

1546 independent reflections

1212 reflections with $I>2 \sigma(I)$

$R_{\text {int }}=0.125$

$\theta_{\text {max }}=25.0^{\circ}, \theta_{\text {min }}=2.1^{\circ}$

$h=-13 \rightarrow 13$

$k=-20 \rightarrow 16$

$l=-5 \rightarrow 3$

$\mathrm{H}$ atoms treated by a mixture of independent and constrained refinement

$w=1 /\left[\sigma^{2}\left(F_{\mathrm{o}}^{2}\right)\right]$

where $P=\left(F_{\mathrm{o}}{ }^{2}+2 F_{\mathrm{c}}{ }^{2}\right) / 3$

$(\Delta / \sigma)_{\max }=0.001$

$\Delta \rho_{\max }=1.64 \mathrm{e} \AA^{-3}$

$\Delta \rho_{\min }=-1.24$ e $\AA^{-3}$

Absolute structure: Flack $x$ determined using 375 quotients $\left[\left(I^{+}\right)-(I)\right] /\left[\left(I^{+}\right)+(I)\right]$ (Parsons et al., 2013)

Absolute structure parameter: -0.02 (5) 


\section{Special details}

Geometry. All esds (except the esd in the dihedral angle between two 1.s. planes) are estimated using the full covariance matrix. The cell esds are taken into account individually in the estimation of esds in distances, angles and torsion angles; correlations between esds in cell parameters are only used when they are defined by crystal symmetry. An approximate (isotropic) treatment of cell esds is used for estimating esds involving l.s. planes.

Fractional atomic coordinates and isotropic or equivalent isotropic displacement parameters $\left(\hat{A}^{2}\right)$

\begin{tabular}{lllll}
\hline & $x$ & $y$ & $z$ & $U_{\mathrm{iso}} * / U_{\mathrm{eq}}$ \\
\hline Cs1 & $0.38524(10)$ & $0.10339(5)$ & $0.6704(2)$ & $0.0179(3)$ \\
Pd1 & $0.28780(11)$ & $0.41984(6)$ & $0.5719(2)$ & $0.0097(3)$ \\
C11 & $0.1180(4)$ & $0.4294(2)$ & $0.8375(9)$ & $0.0184(9)$ \\
C12 & $0.3493(4)$ & $0.5375(2)$ & $0.7574(8)$ & $0.0164(10)$ \\
O1 & $0.2471(9)$ & $0.3141(6)$ & $0.415(2)$ & $0.016(3)$ \\
O2 & $0.3137(9)$ & $0.2136(6)$ & $0.147(2)$ & $0.020(3)$ \\
O3 & $0.5412(9)$ & $0.2649(6)$ & $0.538(2)$ & $0.016(2)$ \\
H3 & $0.6175(15)$ & $0.2695(16)$ & $0.579(8)$ & $0.024^{*}$ \\
N1 & $0.4279(10)$ & $0.4068(7)$ & $0.316(3)$ & $0.014(2)$ \\
H1A & 0.430153 & 0.446008 & 0.178195 & $0.017^{*}$ \\
H1B & 0.492081 & 0.410758 & 0.431882 & $0.017^{*}$ \\
C1 & $0.3228(14)$ & $0.2811(9)$ & $0.245(3)$ & $0.012(4)$ \\
C2 & $0.4286(13)$ & $0.3293(8)$ & $0.158(4)$ & $0.014(2)$ \\
H2 & 0.424782 & 0.339538 & -0.061897 & $0.017^{*}$ \\
C3 & $0.5389(14)$ & $0.2847(9)$ & $0.222(3)$ & $0.016(2)$ \\
H3A & 0.541636 & 0.236400 & 0.099494 & $0.019^{*}$ \\
H3B & 0.606568 & 0.317387 & 0.170155 & $0.019^{*}$ \\
& & & &
\end{tabular}

Atomic displacement parameters $\left(\AA^{2}\right)$

\begin{tabular}{lllllll}
\hline & $U^{11}$ & $U^{22}$ & $U^{33}$ & $U^{12}$ & $U^{13}$ & $U^{23}$ \\
\hline Cs1 & $0.0240(7)$ & $0.0163(5)$ & $0.0134(5)$ & $0.0002(5)$ & $-0.0022(5)$ & $-0.0020(4)$ \\
Pd1 & $0.0094(7)$ & $0.0098(6)$ & $0.0097(6)$ & $0.0012(6)$ & $0.0003(6)$ & $0.0000(5)$ \\
C11 & $0.015(2)$ & $0.0202(19)$ & $0.020(2)$ & $0.001(2)$ & $0.003(2)$ & $-0.0022(17)$ \\
C12 & $0.016(3)$ & $0.0124(19)$ & $0.021(3)$ & $-0.0018(19)$ & $0.0006(18)$ & $-0.0039(15)$ \\
O1 & $0.009(6)$ & $0.015(5)$ & $0.025(7)$ & $-0.003(5)$ & $0.011(5)$ & $-0.005(5)$ \\
O2 & $0.024(8)$ & $0.013(5)$ & $0.024(6)$ & $-0.001(5)$ & $-0.010(6)$ & $-0.003(5)$ \\
O3 & $0.010(5)$ & $0.022(5)$ & $0.016(5)$ & $0.002(4)$ & $0.000(4)$ & $0.002(4)$ \\
N1 & $0.008(5)$ & $0.018(5)$ & $0.017(6)$ & $0.002(4)$ & $-0.004(5)$ & $-0.001(5)$ \\
C1 & $0.008(9)$ & $0.021(9)$ & $0.007(9)$ & $-0.001(7)$ & $0.005(6)$ & $0.003(6)$ \\
C2 & $0.008(5)$ & $0.018(5)$ & $0.017(6)$ & $0.002(4)$ & $-0.004(5)$ & $-0.001(5)$ \\
C3 & $0.010(5)$ & $0.022(5)$ & $0.016(5)$ & $0.002(4)$ & $0.000(4)$ & $0.002(4)$ \\
\hline
\end{tabular}

Geometric parameters $\left(\AA,{ }^{\circ}\right)$

\begin{tabular}{llll}
\hline $\mathrm{Cs} 1-\mathrm{Cs} 1^{\mathrm{i}}$ & $4.421(2)$ & $\mathrm{Pd} 1-\mathrm{O} 1$ & $1.993(9)$ \\
$\mathrm{Cs} 1-\mathrm{Pd} 1^{\mathrm{ii}}$ & $3.8755(17)$ & $\mathrm{Pd} 1-\mathrm{N} 1$ & $2.000(12)$ \\
$\mathrm{Cs} 1-\mathrm{C} 11^{\mathrm{ii}}$ & $3.528(4)$ & $\mathrm{O} 1-\mathrm{C} 1$ & $1.291(17)$ \\
$\mathrm{Cs} 1-\mathrm{C} 11^{\mathrm{iv}}$ & $3.572(4)$ & $\mathrm{O} 2-\mathrm{C} 1$ & $1.238(18)$
\end{tabular}




$$
\begin{aligned}
& \mathrm{Cs} 1-\mathrm{Cl} 1^{\mathrm{ii}} \\
& \mathrm{Cs} 1-\mathrm{Cl} 1^{\mathrm{v}} \\
& \mathrm{Cs} 1-\mathrm{Cl} 2^{\mathrm{ii}} \\
& \mathrm{Cs} 1-\mathrm{Cl} 2^{\mathrm{v}} \\
& \mathrm{Cs} 1-\mathrm{O} 2 \\
& \mathrm{Cs} 1-\mathrm{O} 2^{\mathrm{vi}} \\
& \mathrm{Cs} 1-\mathrm{O} 3 \\
& \mathrm{Cs} 1-\mathrm{C} 1 \\
& \mathrm{Pd} 1-\mathrm{Cl} 1 \\
& \mathrm{Pd} 1-\mathrm{Cl} 2
\end{aligned}
$$

$\mathrm{Pd} 1^{\mathrm{ii}}-\mathrm{Cs} 1-\mathrm{Cs} 1^{\mathrm{i}}$

$\mathrm{Pd} 1^{\mathrm{ii}}-\mathrm{Cs} 1-\mathrm{Cl}^{\mathrm{v}}$

$\mathrm{C} 11^{\mathrm{v}}-\mathrm{Cs} 1-\mathrm{Cs}^{\mathrm{i}}$

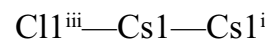

$\mathrm{C} 11^{\mathrm{iv}}-\mathrm{Cs} 1-\mathrm{Cs} 1$

$\mathrm{C} 11^{\mathrm{ii}}-\mathrm{Cs} 1-\mathrm{Cs} 1^{\mathrm{i}}$

Cl1 ${ }^{\mathrm{iv}}-\mathrm{C} 1-\mathrm{Pd} 1^{\mathrm{ii}}$

$\mathrm{C} 11^{\mathrm{iii}}-\mathrm{C} 1-\mathrm{Pd} 1^{\mathrm{ii}}$

$\mathrm{C} 11^{1 i}-\mathrm{Cs} 1-\mathrm{Pd} 1^{\mathrm{ii}}$

$\mathrm{C} 11^{\mathrm{v}}-\mathrm{Cs} 1-\mathrm{Pd} 1^{\mathrm{ii}}$

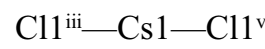

$\mathrm{C} 11^{1 i i}-\mathrm{Cs} 1-\mathrm{Cl1}^{\mathrm{iv}}$

$\mathrm{Cl1}^{\mathrm{iv}}-\mathrm{Cs} 1-\mathrm{Cl1}^{\mathrm{v}}$

Cl1 ${ }^{\text {iii-Cs } 1-C l 1}{ }^{\text {ii }}$

$\mathrm{C} 11^{\mathrm{v}}-\mathrm{Cs} 1-\mathrm{C} 11^{\mathrm{ii}}$

$\mathrm{C} 11^{\text {iv }}-\mathrm{Cs} 1-\mathrm{Cl1}^{1 i}$

$\mathrm{C} 11^{1 i i}-\mathrm{Cs} 1-\mathrm{Cl}^{\mathrm{v}}$

$\mathrm{C} 11^{\mathrm{v}}-\mathrm{Cs} 1-\mathrm{Cl}^{\mathrm{v}}$

$\mathrm{Cl1} 1 \mathrm{ii}-\mathrm{Cs} 1-\mathrm{Cl}^{\mathrm{v}}$

$\mathrm{Cl} 1^{\mathrm{iv}}-\mathrm{Cs} 1-\mathrm{Cl}^{\mathrm{v}}$

$\mathrm{C} 11^{\mathrm{iii}}-\mathrm{C} 1-\mathrm{C} 1$

$\mathrm{C} 11^{\mathrm{iv}}-\mathrm{C}$ s $1-\mathrm{C} 1$

$\mathrm{Cl} 2^{\mathrm{v}}-\mathrm{Cs} 1-\mathrm{Cs}^{\mathrm{i}}$

$\mathrm{Cl} 2^{\mathrm{ii}}-\mathrm{Cs} 1-\mathrm{Cs} 1^{\mathrm{i}}$

$\mathrm{Cl} 2^{\mathrm{ii}}-\mathrm{Cs} 1-\mathrm{Pd} 1^{\mathrm{ii}}$

$\mathrm{C} 22^{\mathrm{ii}}-\mathrm{Cs} 1-\mathrm{C} 11^{\mathrm{iii}}$

$\mathrm{Cl} 2^{\mathrm{ii}}-\mathrm{Cs} 1-\mathrm{Cl}^{\mathrm{v}}$

$\mathrm{Cl} 2 \mathrm{ii}-\mathrm{Cs} 1-\mathrm{C} 11^{\mathrm{ii}}$

$\mathrm{Cl} 2^{\mathrm{ii}}-\mathrm{Cs} 1-\mathrm{Cl}^{\text {iv }}$

$\mathrm{Cl} 2^{\mathrm{ii}}-\mathrm{Cs} 1-\mathrm{Cl}^{\mathrm{v}}$

$\mathrm{Cl} 2$ ii $-\mathrm{Cs} 1-\mathrm{C} 1$

$\mathrm{O} 2-\mathrm{Cs} 1-\mathrm{Cs}^{\mathrm{i}}$

$\mathrm{O} 2^{\mathrm{vi}}-\mathrm{Cs} 1-\mathrm{Cs} 1^{\mathrm{i}}$

$\mathrm{O} 2^{\mathrm{vi}}-\mathrm{Cs} 1-\mathrm{Pd} 1^{1 \mathrm{ii}}$

$\mathrm{O} 2-\mathrm{Cs} 1-\mathrm{Pd} 1^{\mathrm{ii}}$

$\mathrm{O} 2^{\mathrm{vi}}-\mathrm{Cs} 1-\mathrm{C} 11^{\mathrm{iii}}$

$\mathrm{O} 2-\mathrm{Cs} 1-\mathrm{C} 11^{\mathrm{ii}}$
$3.740(4)$

$3.698(4)$

$3.510(4)$

3.901 (4)

$3.117(10)$

$2.962(10)$

$3.349(10)$

$3.654(15)$

2.305 (4)

2.287 (4)

$70.48(4)$

$65.76(6)$

$50.56(7)$

$54.04(6)$

$54.55(6)$

$51.09(7)$

$94.97(7)$

$116.25(7)$

$35.18(7)$

$60.77(7)$

$60.58(12)$

$78.11(9)$

$105.11(6)$

105.13 (6)

73.95 (7)

$59.79(11)$

$94.47(10)$

$50.97(8)$

$99.35(9)$

154.03 (9)

$127.5(3)$

87.0 (3)

$100.91(6)$

$102.14(7)$

$35.65(6)$

151.89 (9)

$93.39(9)$

$53.58(9)$

$100.86(10)$

$74.06(8)$

80.1 (3)

129.96 (19)

$132.5(2)$

124.7 (2)

98.06 (19)

82.3 (2)

$91.2(2)$
$\mathrm{O} 3-\mathrm{H} 3$

$\mathrm{O} 3-\mathrm{C} 3$

N1-H1A

N1-H1B

$\mathrm{N} 1-\mathrm{C} 2$

$\mathrm{C} 1-\mathrm{C} 2$

$\mathrm{C} 2-\mathrm{H} 2$

$\mathrm{C} 2-\mathrm{C} 3$

$\mathrm{C} 3-\mathrm{H} 3 \mathrm{~A}$

$\mathrm{C} 3-\mathrm{H} 3 \mathrm{~B}$

O3-Cs1-C1

$\mathrm{C} 1-\mathrm{Cs} 1-\mathrm{Cs}^{\mathrm{i}}$

$\mathrm{C} 1-\mathrm{Cs} 1-\mathrm{Pd} 1^{\mathrm{ii}}$

$\mathrm{C} 1-\mathrm{Cs} 1-\mathrm{C} 11^{\mathrm{ii}}$

$\mathrm{C} 1-\mathrm{Cs} 1-\mathrm{Cl1}^{\mathrm{v}}$

$\mathrm{C} 1-\mathrm{Cs} 1-\mathrm{Cl}^{\mathrm{v}}$

Cl1-Pd1-Cs1 ${ }^{\text {vii }}$

$\mathrm{Cl} 2-\mathrm{Pd} 1-\mathrm{Cs}^{\mathrm{vii}}$

$\mathrm{Cl} 2-\mathrm{Pd} 1-\mathrm{Cl} 1$

$\mathrm{O} 1-\mathrm{Pd} 1-\mathrm{Cs} 1^{\mathrm{vii}}$

$\mathrm{O} 1-\mathrm{Pd} 1-\mathrm{Cl}$

$\mathrm{O} 1-\mathrm{Pd} 1-\mathrm{Cl} 2$

O1-Pd1-N1

N1-Pd1-Cs1 ${ }^{\text {vii }}$

N1-Pd1-C11

$\mathrm{N} 1-\mathrm{Pd} 1-\mathrm{Cl} 2$

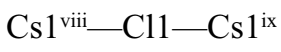

$\mathrm{Cs} 1^{\mathrm{x}}-\mathrm{Cl1}-\mathrm{Cs}^{1}{ }^{\mathrm{ix}}$

$\mathrm{Cs} 1^{\mathrm{ix}}-\mathrm{C} 11-\mathrm{Cs} 1^{\text {vii }}$

$\mathrm{Cs} 1^{\mathrm{x}}-\mathrm{Cl1}-\mathrm{Cs}^{\mathrm{vii}}$

$\mathrm{Cs}^{\text {viii }}-\mathrm{Cl1}-\mathrm{Cs} 1^{\mathrm{vii}}$

$\mathrm{Cs}^{\mathrm{x}}-\mathrm{C} 11-\mathrm{Cs}^{\mathrm{viii}}$

Pd1-Cl1-Cs1 $1^{\text {vii }}$

$\mathrm{Pd} 1-\mathrm{Cl} 1-\mathrm{Cs} 1^{\text {viii }}$

$\mathrm{Pd} 1-\mathrm{Cl1}-\mathrm{Cs}^{\mathrm{x}}$

$\mathrm{Pd} 1-\mathrm{C} 11-\mathrm{Cs} 1^{\mathrm{ix}}$

$\mathrm{Cs} 1^{\mathrm{vii}}-\mathrm{Cl} 2-\mathrm{Cs} 1^{\text {ix }}$

$\mathrm{Pd} 1-\mathrm{Cl} 2-\mathrm{Cs} 1^{\mathrm{ix}}$

$\mathrm{Pd} 1-\mathrm{Cl} 2-\mathrm{Cs} 1^{\text {vii }}$

C1-O1-Pd1

$\mathrm{Cs} 1{ }^{\mathrm{xi}}-\mathrm{O} 2-\mathrm{Cs} 1$

$\mathrm{C} 1-\mathrm{O} 2-\mathrm{Cs} 1^{\mathrm{xi}}$

$\mathrm{C} 1-\mathrm{O} 2-\mathrm{Cs} 1$

$\mathrm{Cs} 1-\mathrm{O} 3-\mathrm{H} 3$

$\mathrm{C} 3-\mathrm{O} 3-\mathrm{Cs} 1$

$\mathrm{C} 3-\mathrm{O} 3-\mathrm{H} 3$

$\mathrm{Pd} 1-\mathrm{N} 1-\mathrm{H} 1 \mathrm{~A}$
$0.907(12)$

1.458 (18)

0.9100

0.9100

$1.500(17)$

$1.53(2)$

1.0000

$1.52(2)$

0.9900

0.9900

48.1 (3)

141.5 (2)

$115.0(2)$

109.9 (2)

$167.2(3)$

$116.3(3)$

$69.20(10)$

63.44 (11)

90.97 (14)

120.7 (3)

92.5 (3)

$175.4(3)$

83.7 (4)

$110.5(3)$

$175.3(4)$

93.0 (3)

119.79 (11)

$75.40(8)$

73.95 (7)

119.82 (11)

$74.36(8)$

78.11 (9)

75.62 (10)

$107.83(14)$

164.55 (15)

111.88 (14)

74.06 (8)

105.91 (14)

80.92 (12)

116.2 (9)

94.8 (3)

145.3 (10)

105.8 (9)

124.3 (19)

110.7 (8)

101 (2)

109.2 


$$
\begin{aligned}
& \mathrm{O} 2-\mathrm{Cs} 1-\mathrm{Cl}^{\text {iv }} \\
& \mathrm{O} 2-\mathrm{Cs} 1-\mathrm{Cl1}^{\mathrm{v}} \\
& \mathrm{O} 2^{\mathrm{vi}}-\mathrm{Cs} 1-\mathrm{Cl}^{1 \mathrm{ii}} \\
& \mathrm{O} 2-\mathrm{Cs} 1-\mathrm{Cl}^{1 \mathrm{ii}} \\
& \mathrm{O} 2^{\mathrm{vi}}-\mathrm{Cs} 1-\mathrm{Cl}^{\mathrm{v}} \\
& \mathrm{O} 2^{\mathrm{vi}}-\mathrm{Cs} 1-\mathrm{Cl}^{\mathrm{iv}} \\
& \mathrm{O} 2^{\mathrm{vi}}-\mathrm{Cs} 1-\mathrm{Cl}_{2}{ }^{\mathrm{ii}} \\
& \mathrm{O} 2-\mathrm{Cs} 1-\mathrm{Cl}^{\mathrm{v}} \\
& \mathrm{O} 2-\mathrm{Cs} 1-\mathrm{Cl}_{2}{ }^{\mathrm{ii}} \\
& \mathrm{O} 2^{\mathrm{vi}}-\mathrm{Cs} 1-\mathrm{Cl}^{\mathrm{v}} \\
& \mathrm{O} 2^{\mathrm{vi}}-\mathrm{Cs} 1-\mathrm{O} 2 \\
& \mathrm{O} 2-\mathrm{Cs} 1-\mathrm{O} 3 \\
& \mathrm{O} 2{ }^{\mathrm{vi}}-\mathrm{Cs} 1-\mathrm{O} 3 \\
& \mathrm{O} 2-\mathrm{Cs} 1-\mathrm{C} 1 \\
& \mathrm{O} 2^{\mathrm{vi}}-\mathrm{Cs} 1-\mathrm{C} 1 \\
& \mathrm{O} 3-\mathrm{Cs} 1-\mathrm{Cs}^{\mathrm{i}} \\
& \mathrm{O} 3-\mathrm{Cs} 1-\mathrm{Pd} 1^{\mathrm{ii}} \\
& \mathrm{O} 3-\mathrm{Cs} 1-\mathrm{Cl}^{\mathrm{iii}} \\
& \mathrm{O} 3-\mathrm{Cs} 1-\mathrm{Cl}^{\mathrm{ii}}
\end{aligned}
$$

$79.5(2)$

$158.4(2)$

$159.9(2)$

$140.2(2)$

$94.5(2)$

$140.3(2)$

$112.3(2)$

$118.8(2)$

$65.0(2)$

$61.0(2)$

$94.8(3)$

$60.9(3)$

$75.8(3)$

$19.0(3)$

$78.0(3)$

$109.42(18)$

$153.60(18)$

80.07 (19)

$123.52(19)$

66.99 (18)

$140.51(19)$

$136.84(18)$

$125.87(19)$

$\begin{array}{ll}\mathrm{Pd} 1-\mathrm{N} 1-\mathrm{H} 1 \mathrm{~B} & 109.2 \\ \mathrm{H} 1 \mathrm{~A}-\mathrm{N} 1-\mathrm{H} 1 \mathrm{~B} & 107.9 \\ \mathrm{C} 2-\mathrm{N} 1-\mathrm{Pd} 1 & 111.9(9) \\ \mathrm{C} 2-\mathrm{N} 1-\mathrm{H} 1 \mathrm{~A} & 109.2 \\ \mathrm{C} 2-\mathrm{N} 1-\mathrm{H} 1 \mathrm{~B} & 109.2 \\ \mathrm{O} 1-\mathrm{C} 1-\mathrm{Cs} 1 & 101.0(8) \\ \mathrm{O} 1-\mathrm{C} 1-\mathrm{C} 2 & 117.5(13) \\ \mathrm{O} 2-\mathrm{C} 1-\mathrm{Cs} 1 & 55.1(8) \\ \mathrm{O} 2-\mathrm{C} 1-\mathrm{O} 1 & 123.8(15) \\ \mathrm{O} 2-\mathrm{C} 1-\mathrm{C} 2 & 118.7(14) \\ \mathrm{C} 2-\mathrm{C} 1-\mathrm{Cs} 1 & 114.9(9) \\ \mathrm{N} 1-\mathrm{C} 2-\mathrm{C} 1 & 110.5(13) \\ \mathrm{N} 1-\mathrm{C} 2-\mathrm{H} 2 & 108.0 \\ \mathrm{~N} 1-\mathrm{C} 2-\mathrm{C} 3 & 111.1(12) \\ \mathrm{C} 1-\mathrm{C} 2-\mathrm{H} 2 & 108.0 \\ \mathrm{C} 3-\mathrm{C} 2-\mathrm{C} 1 & 111.0(13) \\ \mathrm{C} 3-\mathrm{C} 2-\mathrm{H} 2 & 108.0 \\ \mathrm{O} 3-\mathrm{C} 3-\mathrm{C} 2 & 108.4(14) \\ \mathrm{O} 3-\mathrm{C} 3-\mathrm{H} 3 \mathrm{~A} & 110.0 \\ \mathrm{O} 3-\mathrm{C} 3-\mathrm{H} 3 \mathrm{~B} & 110.0 \\ \mathrm{C} 2-\mathrm{C} 3-\mathrm{H} 3 \mathrm{~A} & 110.0 \\ \mathrm{C} 2-\mathrm{C} 3-\mathrm{H} 3 \mathrm{~B} & 110.0 \\ \mathrm{H} 3 \mathrm{~A}-\mathrm{C} 3-\mathrm{H} 3 \mathrm{~B} & 108.4 \\ & \end{array}$

Symmetry codes: (i) $-x+1,-y, z$; (ii) $-x+1 / 2, y-1 / 2,-z+1$; (iii) $x+1 / 2,-y+1 / 2,-z+2$; (iv) $x+1 / 2,-y+1 / 2,-z+1$; (v) $-x+1 / 2, y-1 / 2,-z+2$; (vi) $x, y, z+1$; (vii) $-x+1 / 2, y+1 / 2,-z+1$; (viii) $x-1 / 2,-y+1 / 2,-z+1$; (ix) $-x+1 / 2, y+1 / 2,-z+2$; (x) $x-1 / 2,-y+1 / 2,-z+2$; (xi) $x, y, z-1$.

Hydrogen-bond geometry $\left(A,{ }^{\circ}\right)$

\begin{tabular}{lllll}
\hline$D-\mathrm{H} \cdots A$ & $D-\mathrm{H}$ & $\mathrm{H} \cdots A$ & $D \cdots A$ & $D-\mathrm{H} \cdots A$ \\
\hline $\mathrm{O} 3-\mathrm{H} 3 \cdots \mathrm{O} 1^{\text {iv }}$ & $0.91(1)$ & $2.07(2)$ & $2.750(14)$ & $131(3)$ \\
$\mathrm{O} 3-\mathrm{H} 3 \cdots \mathrm{O} 2^{\text {iv }}$ & $0.91(1)$ & $2.60(2)$ & $3.479(15)$ & $163(3)$ \\
$\mathrm{N} 1-\mathrm{H} 1 A \cdots \mathrm{Cl} 2^{\mathrm{xi}}$ & 0.91 & 2.62 & $3.471(13)$ & 156 \\
$\mathrm{~N} 1-\mathrm{H} 1 B^{\cdots} \cdots \mathrm{Cl}^{\mathrm{xii}}$ & 0.91 & 2.51 & $3.388(13)$ & 163 \\
$\mathrm{C} 2-\mathrm{H} 2 \cdots 3^{\mathrm{xi}}$ & 1.00 & 2.58 & $3.255(19)$ & 125 \\
\hline
\end{tabular}

Symmetry codes: (iv) $x+1 / 2,-y+1 / 2,-z+1$; (xi) $x, y, z-1$; (xii) $-x+1,-y+1, z$. 